\title{
Nearly Kähler six-manifolds with two-torus symmetry
}

\author{
Giovanni Russo and Andrew Swann
}

\begin{abstract}
We consider nearly Kähler 6-manifolds with effective 2-torus symmetry. The multimoment map for the $T^{2}$-action becomes an eigenfunction of the Laplace operator. At regular values, we prove the $T^{2}$-action is necessarily free on the level sets and determines the geometry of three-dimensional quotients. An inverse construction is given locally producing nearly Kähler six-manifolds from three-dimensional data. This is illustrated for structures on the Heisenberg group.
\end{abstract}

\section{Contents}

1 Introduction 1

2 Torus symmetry and multi-moment maps 2

3 Properties of the multi-moment map and regular values 3

4 Reduction to three-manifolds 5

$\begin{array}{lll}5 & \text { Inverse construction } & 7\end{array}$

$6 \quad$ Invariant structures on the Heisenberg group 11

\section{Introduction}

Nearly Kähler manifolds were originally introduced by Gray [5] as follows: let $M$ be an almost Hermitian manifold with Riemannian metric $g$, almost complex structure $J$, and Levi-Civita connection $\nabla$, then $M$ is called nearly Kähler if $\left(\nabla_{X} J\right) X=0$ for each vector field $X$ on $M$. As Nagy showed [10] the important case is that of compact, six-dimensional nearly Kähler manifolds that are not Kähler, and this is what we focus on. In [3], Carrión discusses how the definition above in dimension 6 is related to a system of partial differential equations. Let us define the fundamental two-form $\sigma:=g(J \cdot, \cdot)$. It turns out that Gray's definition is equivalent to the existence of a complex three-form $\psi_{\mathbb{C}}=\psi_{+}+i \psi_{-}$such that

$$
\left\{\begin{array}{l}
\mathrm{d} \sigma=3 \psi_{+} \\
\mathrm{d} \psi_{-}=-2 \sigma \wedge \sigma .
\end{array}\right.
$$

Bär [1] describes the interplay between nearly Kähler 6-manifolds and $\mathrm{G}_{2}$-geometry: let $C=\left(M \times \mathbb{R}_{>0}, g_{C}:=t^{2} g+\mathrm{d} t^{\otimes 2}\right)$ be a seven-dimensional Riemannian cone over a smooth compact manifold $(M, g)$. Assume that the holonomy of the cone is contained in the exceptional compact Lie group $G_{2}$. Then there exists a pair of closed differential forms 
$\varphi$ and $* \varphi$, pointwise equivalent to the model forms on $\mathbb{R}^{7}$. Viewing $M$ as the level set $t=1$ in $C$, the restriction and contraction by $\partial / \partial t$ of $\varphi$ and $* \varphi$ define an $\mathrm{SU}(3)$-structure $\left(\sigma, \psi_{\mathbb{C}}=\psi_{+}+i \psi_{-}\right)$on $M$ such that

$$
\varphi=t^{2} \mathrm{~d} t \wedge \sigma+t^{3} \psi_{+}, \quad * \varphi=\frac{1}{2} t^{4} \sigma \wedge \sigma+t^{3} \psi_{-} \wedge \mathrm{d} t .
$$

The closedness of $\varphi$ and $* \varphi$ is equivalent to $\mathrm{d} \sigma=3 \psi_{+}, \mathrm{d} \psi_{-}=-2 \sigma \wedge \sigma$, so $M$ is nearly Kähler. Since every manifold with holonomy contained in $\mathrm{G}_{2}$ is Ricci-flat, nearly Kähler 6-manifolds are necessarily Einstein with positive scalar curvature. The latter result was actually proved by Gray ([6], Theorem 5.2) before the connection with $\mathrm{G}_{2}$-holonomy had been noticed. In particular, a complete connected nearly Kähler 6-manifold is compact with finite fundamental group, and its universal cover is also a complete nearly Kähler manifold. This is why we can restrict to the connected, simply connected case.

There are a few known examples of nearly Kähler 6-manifolds: Butruille [2] proved that the only homogeneous ones are $\mathbb{S}^{6}, \mathbb{C P}^{3}, F_{1,2}\left(\mathbb{C}^{3}\right)$, and $\mathbb{S}^{3} \times \mathbb{S}^{3}$. Recently, Foscolo and Haskins [4] showed the existence of the first complete inhomogeneous examples by proving there is at least a cohomogeneity one nearly Kähler structure on $\mathbb{S}^{6}$ and $\mathbb{S}^{3} \times \mathbb{S}^{3}$.

The aim of the present work is to lay the foundations for a path to find new examples with weaker symmetry: our starting remark is that the homogeneous cases listed in [2] and the examples in [4] always contain a 2-torus $T^{2}$ as group of symmetries. So we start introducing nearly Kähler 6-manifolds with 2-torus symmetry and construct a multimoment map for the torus action. A remarkable result is that the multi-moment map is an eigenfunction of the Laplace operator (cf. [9]). After discussing some consequences of the latter fact, we use this map to construct a 3 -manifold via $T^{2}$-reduction. Then we describe the inverse process: we get the necessary conditions to construct a principal $T^{2}$-bundle over a 3-manifold, and find the evolution equations that must be satisfied by the geometric structure on its total space in order to get a six-dimensional nearly Kähler manifold. To this purpose, we mimic the construction developed by Madsen and Swann in [8] on the reduction of torsion-free $\mathrm{G}_{2}$-manifolds.

Acknowledgements. We thank Paul-Andi Nagy and Uwe Semmelmann for useful discussions. Both authors are supported by the Danish Council for Independent Research | Natural Sciences Project DFF - 6108-00358, and by the Danish National Research Foundation grant DNRF95 (Centre for Quantum Geometry of Moduli Spaces).

\section{Torus symmetry and multi-moment maps}

Let $M$ be a closed, connected, six-dimensional smooth manifold, and equip it with an $\mathrm{SU}(3)$-structure given by a Riemannian metric $g$, an almost complex structure $J$, isometry of each tangent space, and a complex volume form $\psi_{\mathbb{C}}=\psi_{+}+i \psi_{-}$of type $(3,0)$. Consider the two-form $\sigma:=g(J \cdot, \cdot)$. We say that $\left(M, g, J, \psi_{\mathbb{C}}\right)$ is nearly Kähler if the equations (1.1) are satisfied. From now on we assume that our structure has an effective $T^{2}$-symmetry, so that a 2 -torus acts effectively on $M$ preserving $g, J$ and $\psi_{ \pm}$. Denote by $U$ and $V$ the generating vector fields for the action. Since $T^{2}$ is an Abelian group, we get $[U, V]=0$.

We introduce a multi-moment map for the torus action, adapting the construction of Madsen and Swann [8] to the present case. Let us define the Lie kernel $P_{t^{2}}$ as the $\mathfrak{t}^{2}$-submodule of $\Lambda^{2} \mathfrak{t}^{2}$ given by $P_{\mathfrak{t}^{2}}:=\operatorname{ker}\left(L: \Lambda^{2} \mathfrak{t}^{2} \rightarrow \mathfrak{t}^{2}\right)$, where $L$ is the $\mathfrak{t}^{2}$-linear map induced by the Lie bracket. Since $T^{2}$ is Abelian, $P_{\mathfrak{t}^{2}}=\Lambda^{2} \mathfrak{t}^{2} \cong \mathbb{R}$. Observe that by the first equation in (1.1) the three-form $\psi_{+}$is closed, so the pair $\left(M, 3 \psi_{+}\right)$is a strong geometry (cf. [8]). Now consider the map

$$
\nu_{M}: M \rightarrow P_{\mathfrak{t}^{2}}^{*} \cong \mathbb{R}, \quad \nu_{M}(p):=\sigma_{p}\left(U_{p}, V_{p}\right) .
$$


Recall that the $T^{2}$-action preserves $g$ and $J$, so it preserves $\sigma$, therefore the Lie derivatives $\mathcal{L}_{U} \sigma$ and $\mathcal{L}_{V} \sigma$ vanish. Further, since $U$ and $V$ commute we have $\left.\left.\mathcal{L}_{V}(U\lrcorner \sigma\right)=U\right\lrcorner \mathcal{L}_{V} \sigma=0$. The action preserves $U$ and $V$ as well, so the function $\nu_{M}$ is invariant under the $T^{2}$-action. We can then compute $\mathrm{d} \nu_{M}$ using Cartan's formula:

$$
\begin{aligned}
\left.\left.\mathrm{d} \nu_{M}=\mathrm{d}(V\lrcorner U\right\lrcorner \sigma\right) & \left.\left.\left.=\mathcal{L}_{V}(U\lrcorner \sigma\right)-V\right\lrcorner \mathrm{~d}(U\lrcorner \sigma\right) \\
& \left.\left.=-V\lrcorner \mathcal{L}_{U} \sigma+V\right\lrcorner U\right\lrcorner \mathrm{d} \sigma=3 \psi_{+}(U, V, \cdot) .
\end{aligned}
$$

This shows that $\nu_{M}$ is a multi-moment map for the $T^{2}$-action on $M$.

In what follows we will denote $g(U, U), g(U, V), g(V, V)$ by $g_{U U}, g_{U V}, g_{V V}$ and define a real function $h$ on $M$ satisfying

$$
h^{2}=g_{U U} g_{V V}-g_{U V}^{2}
$$

When there is no danger of confusion we still use the same notation pointwise. Lastly, when we work on the complexified tangent bundle of $M$, we extend $g, J$ and $\psi_{ \pm}$by $\mathbb{C}$-linearity in all their arguments.

Let $\left\{F_{k}, J F_{k}\right\}_{k=1,2,3}$ be an SU(3)-basis of $T_{p} M$ and $\left\{f_{k}, J f_{k}\right\}_{k=1,2,3}$ be its dual. Then, at $p$,

$$
\begin{aligned}
\sigma & =f_{1} \wedge J f_{1}+f_{2} \wedge J f_{2}+f_{3} \wedge J f_{3}, \\
\psi_{\mathbb{C}} & =\left(f_{1}+i J f_{1}\right) \wedge\left(f_{2}+i J f_{2}\right) \wedge\left(f_{3}+i J f_{3}\right) .
\end{aligned}
$$

The volume form splits into real and imaginary parts:

$$
\begin{aligned}
& \psi_{+}=f_{1} \wedge f_{2} \wedge f_{3}-J f_{1} \wedge J f_{2} \wedge f_{3}-f_{1} \wedge J f_{2} \wedge J f_{3}-J f_{1} \wedge f_{2} \wedge J f_{3}, \\
& \psi_{-}=f_{1} \wedge f_{2} \wedge J f_{3}-J f_{1} \wedge J f_{2} \wedge J f_{3}+f_{1} \wedge J f_{2} \wedge f_{3}+J f_{1} \wedge f_{2} \wedge f_{3}
\end{aligned}
$$

Using the action of $\mathrm{SU}(3)$, we can assume without loss of generality that $U_{p}=x_{0} F_{1}$, for some real number $x_{0}$, and $V_{p}=x_{1} F_{1}+y_{1} J F_{1}+x_{2} F_{2}$, for some real numbers $x_{1}, y_{1}, x_{2}$. Since $x_{1}^{2}+y_{1}^{2}+x_{2}^{2}=g_{V V}, \nu_{M}(p)=g\left(J U_{p}, V_{p}\right)$ and $g_{U V}=g_{U U}^{1 / 2} x_{1}$, we get

$$
U_{p}=g_{U U}^{1 / 2} F_{1}, \quad g_{U U}^{1 / 2} V_{p}=\left(g_{U V} F_{1}+\nu_{M} J F_{1}+\left(h^{2}-\nu_{M}^{2}\right)^{1 / 2} F_{2}\right) .
$$

Thus, in general $\mathrm{d} \nu_{M}=3 \psi_{+}(U, V, \cdot)=3\left(h^{2}-\nu_{M}^{2}\right)^{1 / 2} f_{3}$ pointwise. Lastly, observe that

$$
\left.\left.V\lrcorner U\lrcorner(\sigma \wedge \sigma)=2\left(\nu_{M} \sigma-(U\lrcorner \sigma\right) \wedge(V\lrcorner \sigma\right)\right) .
$$

This formula is obtained by expanding the left-hand side.

\section{Properties of the multi-moment map and regular values}

Using the set-up we can then prove the following result:

Lemma 3.1. Let $\Delta$ be the Laplace operator on $C^{\infty}(M)$, defined as $\Delta=\mathrm{d}^{*} \mathrm{~d}:=-* \mathrm{~d} * \mathrm{~d}$. Then

$$
\Delta \nu_{M}=24 \nu_{M}
$$

Proof. Firstly, we show that

$$
* \mathrm{~d} \nu_{M}=*\left(3 \psi_{+}(U, V, \cdot)\right)=\frac{3}{2} \sigma \wedge \sigma \wedge \alpha_{0},
$$


where $\left.\left.\alpha_{0}:=V\right\lrcorner U\right\lrcorner \psi_{-}$. From equations (2.2) and (2.5) we get pointwise

$$
\begin{aligned}
\sigma \wedge \sigma & =2\left(f_{1} \wedge J f_{1} \wedge f_{2} \wedge J f_{2}+f_{1} \wedge J f_{1} \wedge f_{3} \wedge J f_{3}+f_{2} \wedge J f_{2} \wedge f_{3} \wedge J f_{3}\right), \\
\alpha_{0} & =\left(h^{2}-\nu_{M}^{2}\right)^{1 / 2} J f_{3} .
\end{aligned}
$$

Hence $\sigma \wedge \sigma \wedge \alpha_{0}=2\left(h^{2}-\nu_{M}^{2}\right)^{1 / 2} f_{1} \wedge J f_{1} \wedge f_{2} \wedge J f_{2} \wedge J f_{3}$, and

$$
3 \psi_{+}(U, V, \cdot) \wedge\left(\sigma \wedge \sigma \wedge \alpha_{0}\right)=\frac{2}{3}\left\|3 \psi_{+}(U, V, \cdot)\right\|_{g}^{2} \operatorname{vol}_{M}
$$

from which we get (3.2). Thus $\mathrm{d} * \mathrm{~d} \nu_{M}=\frac{3}{2} \sigma \wedge \sigma \wedge \mathrm{d} \alpha_{0}$, since $\mathrm{d}(\sigma \wedge \sigma)=0$. By Cartan's formula $\left.\left.\left.\left.\mathrm{d} \alpha_{0}=\mathrm{d}(V\lrcorner U\right\lrcorner \psi_{-}\right)=-2 V\right\lrcorner U\right\lrcorner(\sigma \wedge \sigma)$. Then, formula (2.6) yields

$$
\begin{aligned}
\mathrm{d} * \mathrm{~d} \nu_{M} & \left.\left.=-6 \nu_{M} \sigma \wedge \sigma \wedge \sigma+6 \sigma \wedge \sigma \wedge(U\lrcorner \sigma\right) \wedge(V\lrcorner \sigma\right) \\
& =-36 \nu_{M} \operatorname{vol}_{M}+12 \nu_{M} \operatorname{vol}_{M} \\
& =-24 \nu_{M} \operatorname{vol}_{M},
\end{aligned}
$$

and we are done.

A consequence of this result is

Proposition 3.2. The average value of the multi-moment map $\nu_{M}$ is 0 . Moreover, the range of $\nu_{M}$ is a compact interval containing 0 in its interior.

Proof. Since $M$ has no boundary we can apply Stokes' theorem and Lemma 3.1:

$$
\begin{aligned}
24 \int_{M} \nu_{M} \operatorname{vol}_{M} & =\int_{M} \Delta \nu_{M} \operatorname{vol}_{M}=\int_{M} \mathrm{~d}^{*} \mathrm{~d} \nu_{M} \wedge * 1=-\int_{M} * \mathrm{~d} * \mathrm{~d} \nu_{M} \wedge * 1 \\
& =\int_{M} \mathrm{~d}\left(* \mathrm{~d} \nu_{M}\right)=\int_{\partial M} * \mathrm{~d} \nu_{M}=0 .
\end{aligned}
$$

So we have our first claim

$$
\int_{M} \nu_{M} \operatorname{vol}_{M}=0
$$

However, $\nu_{M}$ is not constantly zero, and we prove this by contradiction. Assume that $M=\nu_{M}^{-1}(0)$. Then $\sigma(U, V) \equiv 0$, so $V=\eta U+W$ for some real function $\eta$ and some $W$ orthogonal to $U$ and $J U$. Using the action of $\mathrm{SU}(3)$ we can assume pointwise $U=x_{0} F_{1}$, and $W=y_{0} F_{2}$ for some real numbers $x_{0}, y_{0}$. Then $0=\mathrm{d} \nu_{M}=3 \psi_{+}(U, V, \cdot)=3 x_{0} y_{0} f_{3}$. If $U \neq 0$, this implies $y=0$, namely $V=\eta U$. Therefore $V$ and $U$ are linearly dependent pointwise, hence the action of $T^{2}$ is not effective on $M$ ([7], Proposition 4.1), which is a contradiction. This allows us to write $\nu_{M}: M \rightarrow[a, b]$, where $a<0<b$, for $M$ is compact and connected, and $\nu_{M}$ is smooth.

Now assume $s$ is a regular value for $\nu_{M}$. In the next proposition we show that the $T^{2}$-action on $\nu_{M}^{-1}(s)$ is free, so $\nu_{M}^{-1}(s) / T^{2}$ is a smooth 3-manifold and $\nu_{M}^{-1}(s) \rightarrow \nu_{M}^{-1}(s) / T^{2}$ is a principal $T^{2}$-bundle. Its base space is the $T^{2}$-reduction of $M$ at level $s$. We will study the geometry of the quotients $\nu_{M}^{-1}(s) / T^{2}$ in Section 4 .

Proposition 3.3. The multi-moment map $\nu_{M}$ has non-zero regular values. For any regular value $s$ the $T^{2}$-action on $\nu_{M}^{-1}(s)$ is free. Thus $\nu_{M}^{-1}(s) / T^{2}$ are smooth three-dimensional manifolds. 
Proof. By Sard's theorem the set of critical values has Lebesgue measure 0 in $[a, b]$, so we can assert there exist infinitely many regular values for $\nu_{M}$ in $(a, b) \neq \varnothing$. Let $s$ be any of them. Then $\left(\mathrm{d} \nu_{M}\right)_{p}$ has rank 1 for each $p \in \nu_{M}^{-1}(s)$, so $3 \psi_{+}(U, V, \cdot)_{p}=\left(\mathrm{d} \nu_{M}\right)_{p} \neq 0$. Thus $U, V$ are linearly independent over $\mathbb{C}$ on $\nu_{M}^{-1}(s)$, and this yields a locally free action on $\nu_{M}^{-1}(s)$.

On the other hand, if $H$ is the stabilizer in $T^{2}$ of some $p \in \nu_{M}^{-1}(s)$, it preserves $g, J$ and $\psi_{ \pm}$by hypothesis, and also $U$ and $V$. Hence, $H$ fixes $U, J U, V, J V, W:=\psi_{+}(U, V, \cdot)^{\sharp}$ and $J W$, so all of $T_{p} M$. Then, by the Tubular Neighbourhood Theorem, the set $A:=\{p \in$ $M: h \cdot p=p, \mathrm{~d} h_{p}=\operatorname{Id}_{T_{p} M}$ for each $\left.h \in H\right\}$ is open and closed in $M$. Since we assume $M$ connected and $A$ is not empty, $A=M$. If $H$ is not trivial, then we get a contradiction, because the action is effective on $M$. So $H$ must be trivial, and our claim is proved.

\section{Reduction to three-manifolds}

In order to study the geometric structure of the quotients $Q_{s}^{3}:=\nu_{M}^{-1}(s) / T^{2}$, with $s \neq 0$ a regular value for $\nu_{M}$, we need to determine which forms on $\nu_{M}^{-1}(s)$ descend to them. We use the following criterion: a $k$-form $\beta$ on $\nu_{M}^{-1}(s)$ descends to $Q_{s}^{3}$ if and only if it is basic, that is $\mathcal{L}_{U} \beta=\mathcal{L}_{V} \beta=0$ and $\left.\left.U\right\lrcorner \beta=V\right\lrcorner \beta=0$. Before starting, let us define the dual forms of $U$ and $V$ :

$$
\vartheta_{1}:=h^{-2}\left(g_{V V} U^{b}-g_{U V} V^{b}\right), \quad \vartheta_{2}:=h^{-2}\left(g_{U U} V^{b}-g_{U V} U^{b}\right),
$$

where $h$ satisfies (2.1). The pair $\left(\vartheta_{1}, \vartheta_{2}\right)$ is a connection one-form for the $T^{2}$-bundle $\nu_{M}^{-1}(s) \rightarrow \nu_{M}^{-1}(s) / T^{2}$. The invariant functions we find are $g_{U U}, g_{U V}, g_{V V}$. Then we have basic one-forms

$$
\left.\left.\left.\left.\alpha_{0}:=V\right\lrcorner U\right\lrcorner \psi_{-}, \quad \alpha_{1}:=s \vartheta_{1}+V\right\lrcorner \sigma, \quad \alpha_{2}:=s \vartheta_{2}-U\right\lrcorner \sigma,
$$

and lastly the two-forms $\left.U\lrcorner \psi_{+}, V\right\lrcorner \psi_{+}$are basic.

The next step is to specify $g, \sigma, \psi_{ \pm}$on $M$ in terms of the forms $\mathrm{d} \nu_{M}, \vartheta_{1}, \vartheta_{2}, \alpha_{0}, \alpha_{1}, \alpha_{2}$. We work on $M$, pointing out what holds in particular on the level sets $\nu_{M}^{-1}(s)$. Using the basis of the previous section, we find pointwise:

$$
\begin{aligned}
\mathrm{d} \nu_{M} & =3\left(h^{2}-\nu_{M}^{2}\right)^{1 / 2} f_{3}, \\
\vartheta_{1} & =h^{-2} g_{U U}^{-1 / 2}\left(h^{2} f_{1}-g_{U V} \nu_{M} J f_{1}-g_{U V}\left(h^{2}-\nu_{M}^{2}\right)^{1 / 2} f_{2}\right), \\
\vartheta_{2} & =h^{-2} g_{U U}^{1 / 2}\left(\nu_{M} J f_{1}+\left(h^{2}-\nu_{M}^{2}\right)^{1 / 2} f_{2}\right), \\
\alpha_{0} & =\left(h^{2}-\nu_{M}^{2}\right)^{1 / 2} J f_{3}, \\
\alpha_{1} & =h^{-2} g_{U U}^{-1 / 2}\left(h^{2}-\nu_{M}^{2}\right)^{1 / 2}\left(g_{U V}\left(h^{2}-\nu_{M}^{2}\right)^{1 / 2} J f_{1}-g_{U V} \nu_{M} f_{2}+h^{2} J f_{2}\right), \\
\alpha_{2} & =h^{-2} g_{U U}^{1 / 2}\left(h^{2}-\nu_{M}^{2}\right)^{1 / 2}\left(\nu_{M} f_{2}-\left(h^{2}-\nu_{M}^{2}\right)^{1 / 2} J f_{1}\right) .
\end{aligned}
$$

Now let $s \neq 0$. The last five forms are linearly independent on $\nu_{M}^{-1}(s)$ if and only if $h^{2} \neq s^{2}$. Observe that $h^{2}-\nu_{M}^{2}=\left\|\left(h^{2}-\nu_{M}^{2}\right)^{1 / 2} f_{3}\right\|_{g}^{2}=\frac{1}{9}\left\|\mathrm{~d} \nu_{M}\right\|_{g}^{2}=\left\|\psi_{+}(U, V, \cdot)\right\|_{g}^{2}$, and this quantity is non-zero under our assumptions. Therefore, we can compute the general expressions of 
$g, \sigma, \psi_{ \pm}$on $M:$

$$
\begin{aligned}
g= & \frac{1}{9\left(h^{2}-\nu_{M}^{2}\right)} \mathrm{d} \nu_{M}^{\otimes 2}+g_{U U} \vartheta_{1}^{\otimes 2}+g_{V V} \vartheta_{2}^{\otimes 2}+g_{U V}\left(\vartheta_{1} \otimes \vartheta_{2}+\vartheta_{2} \otimes \vartheta_{1}\right) \\
& +\frac{1}{h^{2}-\nu_{M}^{2}}\left(\alpha_{0}^{\otimes 2}+g_{U U} \alpha_{1}^{\otimes 2}+g_{V V} \alpha_{2}^{\otimes 2}+g_{U V}\left(\alpha_{1} \otimes \alpha_{2}+\alpha_{2} \otimes \alpha_{1}\right)\right) ; \\
\sigma= & \frac{1}{3\left(h^{2}-\nu_{M}^{2}\right)} \mathrm{d} \nu_{M} \wedge \alpha_{0}+\nu_{M} \vartheta_{1} \wedge \vartheta_{2}-\vartheta_{1} \wedge \alpha_{2}+\vartheta_{2} \wedge \alpha_{1}-\frac{\nu_{M}}{h^{2}-\nu_{M}^{2}} \alpha_{1} \wedge \alpha_{2} ; \\
\psi_{+}= & \frac{1}{3\left(h^{2}-\nu_{M}^{2}\right)} \mathrm{d} \nu_{M} \wedge\left(\left(h^{2}-\nu_{M}^{2}\right) \vartheta_{1} \wedge \vartheta_{2}+\nu_{M}\left(\vartheta_{1} \wedge \alpha_{2}-\vartheta_{2} \wedge \alpha_{1}\right)-\alpha_{1} \wedge \alpha_{2}\right) \\
& -\frac{1}{h^{2}-\nu_{M}^{2}}\left(\vartheta_{1} \wedge\left(g_{U U} \alpha_{1}+g_{U V} \alpha_{2}\right)+\vartheta_{2} \wedge\left(g_{U V} \alpha_{1}+g_{V V} \alpha_{2}\right)\right) \wedge \alpha_{0} ; \\
\psi_{-}= & \frac{1}{3\left(h^{2}-\nu_{M}^{2}\right)} \mathrm{d} \nu_{M} \wedge\left(\vartheta_{1} \wedge\left(g_{U U} \alpha_{1}+g_{U V} \alpha_{2}\right)+\vartheta_{2} \wedge\left(g_{U V} \alpha_{1}+g_{V V} \alpha_{2}\right)\right) \\
& +\frac{1}{h^{2}-\nu_{M}^{2}}\left(\left(h^{2}-\nu_{M}^{2}\right) \vartheta_{1} \wedge \vartheta_{2}+\nu_{M}\left(\vartheta_{1} \wedge \alpha_{2}-\vartheta_{2} \wedge \alpha_{1}\right)-\alpha_{1} \wedge \alpha_{2}\right) \wedge \alpha_{0} .
\end{aligned}
$$

If we use the nearly Kähler structure equations (1.1) we get further relationships. The cotangent space of $M$ splits as the direct sum $V \oplus H$, where $\vartheta_{i} \in V, i=1,2$ and $H$ contains $\mathrm{d} \nu_{M}, \alpha_{k}, k=0,1,2$. Comparing coefficients in $\mathrm{d} \sigma=3 \psi_{+}$we obtain

$$
\begin{gathered}
\nu_{M} \mathrm{~d} \vartheta_{2}=\mathrm{d} \alpha_{2}+\frac{1}{h^{2}-\nu_{M}^{2}}\left(3 g_{U U} \alpha_{1} \wedge \alpha_{0}+3 g_{U V} \alpha_{2} \wedge \alpha_{0}+\nu_{M} \mathrm{~d} \nu_{M} \wedge \alpha_{2}\right), \\
\nu_{M} \mathrm{~d} \vartheta_{1}=\mathrm{d} \alpha_{1}-\frac{1}{h^{2}-\nu_{M}^{2}}\left(3 g_{U V} \alpha_{1} \wedge \alpha_{0}+3 g_{V V} \alpha_{2} \wedge \alpha_{0}-\nu_{M} \mathrm{~d} \nu_{M} \wedge \alpha_{1}\right) \\
\mathrm{d} \vartheta_{1} \wedge \alpha_{2}-\mathrm{d} \vartheta_{2} \wedge \alpha_{1}=\left(\frac{2 \nu_{M}^{2}}{\left(h^{2}-\nu_{M}^{2}\right)^{2}} \mathrm{~d} \nu_{M}-\frac{\nu_{M}}{\left(h^{2}-\nu_{M}^{2}\right)^{2}} \mathrm{~d}\left(h^{2}\right)\right) \wedge \alpha_{2} \wedge \alpha_{1} \\
+\mathrm{d} \nu_{M} \wedge\left(\frac{1}{3\left(h^{2}-\nu_{M}^{2}\right)^{2}} \mathrm{~d}\left(h^{2}\right) \wedge \alpha_{0}-\frac{1}{3\left(h^{2}-\nu_{M}^{2}\right)} \mathrm{d} \alpha_{0}\right) \\
+\frac{\nu_{M}}{h^{2}-\nu_{M}^{2}} \mathrm{~d}\left(\alpha_{2} \wedge \alpha_{1}\right)
\end{gathered}
$$

The equation $\mathrm{d} \psi_{-}=-2 \sigma \wedge \sigma$ gives

$$
\mathrm{d} \alpha_{0}=-\frac{4 \nu_{M}}{3\left(h^{2}-\nu_{M}^{2}\right)} \mathrm{d} \nu_{M} \wedge \alpha_{0}+\frac{4 h^{2}}{h^{2}-\nu_{M}^{2}} \alpha_{1} \wedge \alpha_{2}
$$




$$
\begin{aligned}
\mathrm{d} \vartheta_{2} \wedge \alpha_{0}= & \frac{-1}{3\left(h^{2}-\nu_{M}^{2}\right)^{2}}\left(\mathrm{~d} \nu_{M} \wedge\left(g_{U U} \alpha_{1}+g_{U V} \alpha_{2}\right)+3 \nu_{M} \alpha_{0} \wedge \alpha_{2}\right) \wedge \mathrm{d}\left(h^{2}\right) \\
& -\frac{1}{3\left(h^{2}-\nu_{M}^{2}\right)}\left(\mathrm{d} \nu_{M} \wedge \mathrm{d}\left(g_{U U} \alpha_{1}+g_{U V} \alpha_{2}\right)+3 \nu_{M} \mathrm{~d} \alpha_{2} \wedge \alpha_{0}\right) \\
& \quad-\frac{h^{2}-3 \nu_{M}^{2}}{3\left(h^{2}-\nu_{M}^{2}\right)^{2}} \mathrm{~d} \nu_{M} \wedge \alpha_{0} \wedge \alpha_{2}, \\
\mathrm{~d} \vartheta_{1} \wedge \alpha_{0}= & \frac{1}{3\left(h^{2}-\nu_{M}^{2}\right)^{2}}\left(\mathrm{~d} \nu_{M} \wedge\left(g_{U V} \alpha_{1}+g_{V V} \alpha_{2}\right)-3 \nu_{M} \alpha_{0} \wedge \alpha_{1}\right) \wedge \mathrm{d}\left(h^{2}\right) \\
& +\frac{1}{3\left(h^{2}-\nu_{M}^{2}\right)}\left(\mathrm{d} \nu_{M} \wedge \mathrm{d}\left(g_{U V} \alpha_{1}+g_{V V} \alpha_{2}\right)-3 \nu_{M} \mathrm{~d} \alpha_{1} \wedge \alpha_{0}\right) \\
& -\frac{h^{2}-3 \nu_{M}^{2}}{3\left(h^{2}-\nu_{M}^{2}\right)^{2}} \mathrm{~d} \nu_{M} \wedge \alpha_{0} \wedge \alpha_{1} .
\end{aligned}
$$

The relations among $\alpha_{0}, \alpha_{1}, \alpha_{2}$ on the $T^{2}$-reduction at level $s$ are then

$$
\begin{aligned}
\mathrm{d} \alpha_{0} & =\frac{4 h^{2}}{h^{2}-s^{2}} \alpha_{1} \wedge \alpha_{2}, \\
\mathrm{~d} \alpha_{1} \wedge \alpha_{0} & =\frac{s^{2}}{h^{2}\left(h^{2}-s^{2}\right)} \mathrm{d}\left(h^{2}\right) \wedge \alpha_{1} \wedge \alpha_{0}, \\
\mathrm{~d} \alpha_{2} \wedge \alpha_{0} & =\frac{s^{2}}{h^{2}\left(h^{2}-s^{2}\right)} \mathrm{d}\left(h^{2}\right) \wedge \alpha_{2} \wedge \alpha_{0} .
\end{aligned}
$$

Define $f:=4 h^{2} /\left(h^{2}-s^{2}\right)$. Observe that $f>4$, and

$$
\frac{\mathrm{d} f}{f}=-\frac{s^{2}}{h^{2}\left(h^{2}-s^{2}\right)} \mathrm{d}\left(h^{2}\right) .
$$

Hence we can summarise our results as follows.

Proposition 4.1. On the level sets $\nu_{M}^{-1}(s)$, with $s \neq 0$ regular value for $\nu_{M}$, we get

$$
\begin{aligned}
& s \mathrm{~d} \vartheta_{1}=\mathrm{d} \alpha_{1}-\frac{1}{h^{2}-s^{2}}\left(3 g_{U V} \alpha_{1}+3 g_{V V} \alpha_{2}\right) \wedge \alpha_{0}, \\
& s \mathrm{~d} \vartheta_{2}=\mathrm{d} \alpha_{2}+\frac{1}{h^{2}-s^{2}}\left(3 g_{U U} \alpha_{1}+3 g_{U V} \alpha_{2}\right) \wedge \alpha_{0} .
\end{aligned}
$$

Proposition 4.2. Define $f:=4 h^{2} /\left(h^{2}-s^{2}\right)$. The relations among $\alpha_{0}, \alpha_{1}, \alpha_{2}$ on the $T^{2}$-reduction at level $s \neq 0$ are given by

$$
\mathrm{d} \alpha_{0}=f \alpha_{1} \wedge \alpha_{2}, \quad \mathrm{~d} \alpha_{1} \wedge \alpha_{0}=-\frac{\mathrm{d} f}{f} \wedge \alpha_{1} \wedge \alpha_{0}, \quad \mathrm{~d} \alpha_{2} \wedge \alpha_{0}=-\frac{\mathrm{d} f}{f} \wedge \alpha_{2} \wedge \alpha_{0} .
$$

Remark 4.3. Define $\beta_{0}=\alpha_{0}$ and $\beta_{i}=f \alpha_{i}, i=1,2$. The equations in (4.16) are then equivalent to

$$
\mathrm{d} \beta_{0}=\frac{1}{f} \beta_{1} \wedge \beta_{2}, \quad \mathrm{~d} \beta_{1} \wedge \beta_{0}=0, \quad \mathrm{~d} \beta_{2} \wedge \beta_{0}=0
$$

\section{Inverse construction}

Now we wish to invert the construction described so far. Assume we are given a threedimensional smooth manifold $Q^{3}$, and let $g_{U U}, g_{U V}, g_{V V}$ be three $s$-dependent functions 
on $Q^{3}$ such that $g_{U U}>0$ and $g_{U U} g_{V V}-g_{U V}^{2}>0$. We define the latter quantity as $h^{2}:=g_{U U} g_{V V}-g_{U V}^{2}$. Let $f>4$ be a real function and $\alpha_{0}, \alpha_{1}, \alpha_{2}$ be a basis of $s$-dependent one-forms satisfying (4.16). Our first goal is to construct a principal $T^{2}$-bundle over $Q^{3}$. Let

$$
\begin{aligned}
& \Theta_{1}:=\frac{1}{s}\left(\mathrm{~d} \alpha_{1}-\frac{3}{h^{2}-s^{2}}\left(g_{U V} \alpha_{1}+g_{V V} \alpha_{2}\right) \wedge \alpha_{0}\right), \\
& \Theta_{2}:=\frac{1}{s}\left(\mathrm{~d} \alpha_{2}+\frac{3}{h^{2}-s^{2}}\left(g_{U U} \alpha_{1}+g_{U V} \alpha_{2}\right) \wedge \alpha_{0}\right),
\end{aligned}
$$

and find the conditions for which they are closed and with integral period, namely $\left[\Theta_{i}\right] \in$ $H^{2}\left(Q_{s}^{3}, \mathbb{Z}\right)$. We apply Proposition 2.3, [11], for this last part. If

$$
\mathrm{d} \Theta_{1}=0=\mathrm{d}\left(\frac{3}{h^{2}-s^{2}}\left(g_{U V} \alpha_{1}+g_{V V} \alpha_{2}\right) \wedge \alpha_{0}\right)
$$

we get

$$
\frac{g_{U V}}{h^{2}} \mathrm{~d}\left(h^{2}\right) \wedge \alpha_{1} \wedge \alpha_{0}+\frac{g_{V V}}{h^{2}} \mathrm{~d}\left(h^{2}\right) \wedge \alpha_{2} \wedge \alpha_{0}=\mathrm{d} g_{U V} \wedge \alpha_{1} \wedge \alpha_{0}+\mathrm{d} g_{V V} \wedge \alpha_{2} \wedge \alpha_{0}
$$

Similarly $\mathrm{d} \Theta_{2}=0$ yields

$$
\frac{g_{U U}}{h^{2}} \mathrm{~d}\left(h^{2}\right) \wedge \alpha_{1} \wedge \alpha_{0}+\frac{g_{U V}}{h^{2}} \mathrm{~d}\left(h^{2}\right) \wedge \alpha_{2} \wedge \alpha_{0}=\mathrm{d} g_{U U} \wedge \alpha_{1} \wedge \alpha_{0}+\mathrm{d} g_{U V} \wedge \alpha_{2} \wedge \alpha_{0} .
$$

Under the conditions (5.3) and (5.4) one can apply the same process as in Paragraph 2.1, [11]: we construct a principal $T^{2}$-bundle $E^{5} \rightarrow Q^{3}$ with $s$-dependent connection one-forms $\vartheta_{1}$ and $\vartheta_{2}$ such that $\mathrm{d} \vartheta_{k}=\Theta_{k}, k=1,2$. The space $E^{5}$ must be thought of as the level set $\nu_{M}^{-1}(s)$ of the previous section. So as $s$ varies we have a five-dimensional foliation of a six-dimensional manifold $M=E \times(c, d)$, for some real numbers $c<d$. To construct a nearly Kähler structure on $M$ starting from $E^{5}$, we flow the $\vartheta_{k}$ 's and the $\alpha_{i}$ 's along the normal vector field to $E^{5}$, given by $\partial / \partial s=\left(9\left(h^{2}-s^{2}\right)\right)^{-1} \mathrm{~d} s^{\sharp}$.

In order to establish which equations must be satisfied, we first define $\sigma, \psi_{ \pm}$as in (4.2)(4.4), then impose the nearly Kähler conditions as we have done above, getting (4.5)-(4.10). Notice that on $M$, the differential d can be split as the sum of the differential on $E^{5}$ and the one in the remaining direction: we write $\mathrm{d}_{6}=\mathrm{d}_{5}+\mathrm{d}_{s}$, where $\mathrm{d}_{5}$ is the differential on $E^{5}$ and $\mathrm{d}_{s} \gamma=\gamma^{\prime} \wedge \mathrm{d} s$, the prime denoting the derivative with respect to $s$ of the form $\gamma$. We use this on (4.5)-(4.10) and then contract with $\partial / \partial s$. The equations found will tell us how our forms evolve in the direction defined by $\partial / \partial s$.

We have seen that $\mathrm{d} \sigma=3 \psi_{+}$implies (4.6) in particular. We rewrite this equation as

$$
\begin{aligned}
s \mathrm{~d}_{5} \vartheta_{1}+s \mathrm{~d}_{s} \vartheta_{1}= & \mathrm{d}_{3} \alpha_{1}+\mathrm{d}_{s} \alpha_{1} \\
& -\frac{1}{h^{2}-s^{2}}\left(3 g_{U V} \alpha_{1} \wedge \alpha_{0}+3 g_{V V} \alpha_{2} \wedge \alpha_{0}+s \alpha_{1} \wedge \mathrm{d} \nu_{M}\right) .
\end{aligned}
$$

By assumption, on $E^{5}$ we have

$$
s \mathrm{~d}_{5} \vartheta_{1}=\mathrm{d}_{3} \alpha_{1}-\frac{1}{h^{2}-s^{2}}\left(3 g_{U V} \alpha_{1}+3 g_{V V} \alpha_{2}\right) \wedge \alpha_{0},
$$

so we can simplify our equation getting

$$
\vartheta_{1}^{\prime} \wedge \mathrm{d} s=\frac{1}{s} \alpha_{1}^{\prime} \wedge \mathrm{d} s-\frac{1}{h^{2}-s^{2}} \alpha_{1} \wedge \mathrm{d} s .
$$


Contracting with $\partial / \partial s$, we obtain

$$
\vartheta_{1}^{\prime}=\frac{1}{s} \alpha_{1}^{\prime}-\frac{1}{h^{2}-s^{2}} \alpha_{1} .
$$

Similarly, from (4.5) we have

$$
\vartheta_{2}^{\prime}=\frac{1}{s} \alpha_{2}^{\prime}-\frac{1}{h^{2}-s^{2}} \alpha_{2} .
$$

From (4.7) and (4.8) we get

$$
\begin{aligned}
\vartheta_{2}^{\prime} \wedge \alpha_{1}-\vartheta_{1}^{\prime} \wedge \alpha_{2}=\frac{-4 h^{2}-6 s^{2}+3 s\left(h^{2}\right)^{\prime}}{3\left(h^{2}-s^{2}\right)^{2}} \alpha_{1} \wedge \alpha_{2} \\
+\frac{s}{h^{2}-s^{2}}\left(\alpha_{2} \wedge \alpha_{1}\right)^{\prime}+\frac{1}{3\left(h^{2}-s^{2}\right)^{2}} \mathrm{~d}_{3}\left(h^{2}\right) \wedge \alpha_{0},
\end{aligned}
$$

and by (4.8) itself we find

$$
\alpha_{0}^{\prime}=\frac{4 s}{3\left(h^{2}-s^{2}\right)} \alpha_{0} .
$$

The remaining equations yield

$$
\begin{aligned}
\alpha_{0} \wedge \vartheta_{2}^{\prime}= & \frac{s}{\left(h^{2}-s^{2}\right)^{2}}\left(h^{2}\right)^{\prime} \alpha_{2} \wedge \alpha_{0}+\frac{1}{3\left(h^{2}-s^{2}\right)^{2}} \mathrm{~d}_{3}\left(h^{2}\right) \wedge\left(g_{U U} \alpha_{1}+g_{U V} \alpha_{2}\right) \\
& \quad+\frac{s}{h^{2}-s^{2}} \alpha_{2}^{\prime} \wedge \alpha_{0}+\frac{h^{2}-3 s^{2}}{3\left(h^{2}-s^{2}\right)^{2}} \alpha_{2} \wedge \alpha_{0}-\frac{1}{3\left(h^{2}-s^{2}\right)} \mathrm{d}_{3}\left(g_{U U} \alpha_{1}+g_{U V} \alpha_{2}\right), \\
\alpha_{0} \wedge \vartheta_{1}^{\prime}= & \frac{s}{\left(h^{2}-s^{2}\right)^{2}}\left(h^{2}\right)^{\prime} \alpha_{1} \wedge \alpha_{0}-\frac{1}{3\left(h^{2}-s^{2}\right)^{2}} \mathrm{~d}_{3}\left(h^{2}\right) \wedge\left(g_{U V} \alpha_{1}+g_{V V} \alpha_{2}\right) \\
& \quad+\frac{s}{h^{2}-s^{2}} \alpha_{1}^{\prime} \wedge \alpha_{0}+\frac{h^{2}-3 s^{2}}{3\left(h^{2}-s^{2}\right)^{2}} \alpha_{1} \wedge \alpha_{0}+\frac{1}{3\left(h^{2}-s^{2}\right)} \mathrm{d}_{3}\left(g_{U V} \alpha_{1}+g_{V V} \alpha_{2}\right) .
\end{aligned}
$$

If we set $\alpha_{1}^{\prime}=\sum_{i} a_{1 i} \alpha_{i}, \alpha_{2}^{\prime}=\sum_{j} a_{2 j} \alpha_{j}$, and

$$
\mathrm{d}_{3} \alpha_{1}=\sum_{i<j} b_{i j} \alpha_{i} \wedge \alpha_{j}, \quad \mathrm{~d}_{3} \alpha_{2}=\sum_{i<j} c_{i j} \alpha_{i} \wedge \alpha_{j},
$$

we can find equations giving $a_{10}, a_{11}, a_{12}, a_{20}, a_{21}, a_{22}, g_{U U}^{\prime}, g_{U V}^{\prime}, g_{V V}^{\prime},\left(h^{2}\right)^{\prime}$. Denote by $X_{i}$ the dual of $\alpha_{i}$. Using (5.5) and (5.6) in (5.7), (5.9) and (5.10), we get

$$
\begin{aligned}
\left(\alpha_{2} \wedge \alpha_{1}\right)^{\prime}=- & \frac{s^{2}}{h^{2}\left(h^{2}-s^{2}\right)}\left(h^{2}\right)^{\prime} \alpha_{1} \wedge \alpha_{2}+\frac{10 s}{3\left(h^{2}-s^{2}\right)} \alpha_{1} \wedge \alpha_{2}-\frac{s}{3 h^{2}\left(h^{2}-s^{2}\right)} \mathrm{d}_{3}\left(h^{2}\right) \wedge \alpha_{0}, \\
\alpha_{0} \wedge \alpha_{2}^{\prime}=- & \frac{s^{2}}{h^{2}\left(h^{2}-s^{2}\right)}\left(h^{2}\right)^{\prime} \alpha_{0} \wedge \alpha_{2}+\frac{2 s}{3\left(h^{2}-s^{2}\right)} \alpha_{0} \wedge \alpha_{2} \\
& +\frac{s}{3 h^{2}\left(h^{2}-s^{2}\right)} \mathrm{d}_{3}\left(h^{2}\right) \wedge\left(g_{U U} \alpha_{1}+g_{U V} \alpha_{2}\right)-\frac{s}{3 h^{2}} \mathrm{~d}_{3}\left(g_{U U} \alpha_{1}+g_{U V} \alpha_{2}\right), \\
\alpha_{0} \wedge \alpha_{1}^{\prime}=- & \frac{s^{2}}{h^{2}\left(h^{2}-s^{2}\right)}\left(h^{2}\right)^{\prime} \alpha_{0} \wedge \alpha_{1}+\frac{2 s}{3\left(h^{2}-s^{2}\right)} \alpha_{0} \wedge \alpha_{1} \\
& \quad-\frac{s}{3 h^{2}\left(h^{2}-s^{2}\right)} \mathrm{d}_{3}\left(h^{2}\right) \wedge\left(g_{U V} \alpha_{1}+g_{V V} \alpha_{2}\right)+\frac{s}{3 h^{2}} \mathrm{~d}_{3}\left(g_{U V} \alpha_{1}+g_{V V} \alpha_{2}\right) .
\end{aligned}
$$


From these equations, comparing the coefficients of $\alpha_{0} \wedge \alpha_{1}, \alpha_{0} \wedge \alpha_{2}$ and $\alpha_{1} \wedge \alpha_{2}$, we find

$$
\begin{aligned}
a_{10} & =\frac{s}{3 h^{2}\left(h^{2}-s^{2}\right)} X_{2}\left(h^{2}\right), \quad a_{20}=\frac{-s}{3 h^{2}\left(h^{2}-s^{2}\right)} X_{1}\left(h^{2}\right), \\
a_{21} & =\frac{g_{U U} s}{3 h^{2}\left(h^{2}-s^{2}\right)} X_{0}\left(h^{2}\right)-\frac{s}{3 h^{2}}\left(X_{0}\left(g_{U U}\right)+g_{U U} b_{01}+g_{U V} c_{01}\right), \\
a_{12} & =\frac{-g_{V V} s}{3 h^{2}\left(h^{2}-s^{2}\right)} X_{0}\left(h^{2}\right)+\frac{s}{3 h^{2}}\left(X_{0}\left(g_{V V}\right)+g_{U V} b_{02}+g_{V V} c_{02}\right), \\
\left(h^{2}\right)^{\prime} & =-\frac{2 h^{2}}{s}+\frac{h^{2}-s^{2}}{3 s}\left(\left(b_{01}-c_{02}\right) g_{U V}+c_{01} g_{V V}-b_{02} g_{U U}\right), \\
a_{11} & =\frac{8 s}{3\left(h^{2}-s^{2}\right)}+\frac{s}{3 h^{2}}\left(X_{0}\left(g_{U V}\right)+b_{02} g_{U U}+c_{02} g_{U V}\right)-\frac{s g_{U V}}{3 h^{2}\left(h^{2}-s^{2}\right)} X_{0}\left(h^{2}\right), \\
a_{22} & =\frac{8 s}{3\left(h^{2}-s^{2}\right)}-\frac{s}{3 h^{2}}\left(X_{0}\left(g_{U V}\right)+b_{01} g_{U V}+c_{01} g_{V V}\right)+\frac{s g_{U V}}{3 h^{2}\left(h^{2}-s^{2}\right)} X_{0}\left(h^{2}\right) .
\end{aligned}
$$

Further, differentiating (4.5), (4.6), and repeating the same process, we get

$$
\begin{aligned}
& g_{U U}^{\prime}=g_{U U}\left(\frac{\left(h^{2}\right)^{\prime}}{h^{2}-s^{2}}+\frac{1}{s}+a_{11}-\frac{2 s}{3\left(h^{2}-s^{2}\right)}\right)-\frac{h^{2}}{3 s} c_{01}+g_{U V} a_{21} \\
& g_{U V}^{\prime}=g_{U V}\left(\frac{\left(h^{2}\right)^{\prime}}{h^{2}-s^{2}}+\frac{1}{s}+a_{22}-\frac{2 s}{3\left(h^{2}-s^{2}\right)}\right)-\frac{h^{2}}{3 s} c_{02}+g_{U U} a_{12}+\frac{s X_{0}\left(h^{2}\right)}{3\left(h^{2}-s^{2}\right)} \\
& g_{U V}^{\prime}=g_{U V}\left(\frac{\left(h^{2}\right)^{\prime}}{h^{2}-s^{2}}+\frac{1}{s}+a_{11}-\frac{2 s}{3\left(h^{2}-s^{2}\right)}\right)+\frac{h^{2}}{3 s} b_{01}+g_{V V} a_{21}-\frac{s X_{0}\left(h^{2}\right)}{3\left(h^{2}-s^{2}\right)} \\
& g_{V V}^{\prime}=g_{V V}\left(\frac{\left(h^{2}\right)^{\prime}}{h^{2}-s^{2}}+\frac{1}{s}+a_{22}-\frac{2 s}{3\left(h^{2}-s^{2}\right)}\right)+\frac{h^{2}}{3 s} b_{02}+g_{U V} a_{12} .
\end{aligned}
$$

These results imply that $\alpha_{0}, \alpha_{1}, \alpha_{2}, g_{U U}, g_{U V}, g_{V V}$ can be found from a system of first order ordinary differential equations, so by Cauchy theorem we find a unique local solution on $E^{5} \times\left(s_{0}-\varepsilon, s_{0}+\varepsilon\right)$, for some $\varepsilon>0$, where $s_{0} \neq 0$ is an initial data. Observe that the value of $s_{0}$ is specified by $f$ and $h$ through the equation $f=4 h^{2} /\left(h^{2}-s_{0}^{2}\right)$. Finally, (5.5) and (5.6), together with the expressions of $\alpha_{1}^{\prime}$ and $\alpha_{2}^{\prime}$ found, give differential equations for $\vartheta_{1}, \vartheta_{2}$, to which we can apply the same theorem. Thus we have the final result:

Theorem 5.1. Let $Q^{3}$ be a smooth 3-manifold, $f>4$ a smooth real function on $Q^{3}$, and $\left\{\alpha_{i}\right\}_{i=0,1,2}$ a basis of one-forms on $Q^{3}$ satisfying (4.16). Suppose there exists a smooth positive definite $G=\left(\begin{array}{ll}g_{U U} & g_{U V} \\ g_{U V} & g_{V V}\end{array}\right)$ on $Q^{3}$ such that (5.3) and (5.4) are fulfilled, and that $s=s_{0}=(1-4 / f)^{1 / 2} h$ is constant. Put $h^{2}=\operatorname{det} G$, and define $\Theta_{1}, \Theta_{2}$ by (5.1) and (5.2) for $s=s_{0}$.

Then, if $\Theta_{k}$ 's have integral periods, there exist a $T^{2}$-bundle $E^{5} \rightarrow Q^{3}$ with connection one-form $\left(\vartheta_{1}, \vartheta_{2}\right)$, such that $\mathrm{d} \vartheta_{k}=\Theta_{k}$, and an $\varepsilon>0$ such that $E^{5} \times\left(s_{0}-\varepsilon, s_{0}+\varepsilon\right)$ has a unique nearly Kähler structure of the form (4.2)-(4.4).

Proof. Let ' denote differentiation with respect to $s$ and assume the functions $a_{i j}$ 's are 
those listed in (5.11)-(5.16). Then our forms satisfy the equations

$$
\begin{gathered}
\alpha_{0}^{\prime}=\frac{4 s}{3\left(h^{2}-s^{2}\right)} \alpha_{0}, \quad \alpha_{1}^{\prime}=\sum_{i=0}^{2} a_{1 i} \alpha_{i}, \quad \alpha_{2}^{\prime}=\sum_{j=0}^{2} a_{2 j} \alpha_{j}, \\
\vartheta_{1}^{\prime}=\frac{1}{s} \alpha_{1}^{\prime}-\frac{1}{h^{2}-s^{2}} \alpha_{1}, \quad \vartheta_{2}^{\prime}=\frac{1}{s} \alpha_{2}^{\prime}-\frac{1}{h^{2}-s^{2}} \alpha_{2} .
\end{gathered}
$$

For the initial data $s=s_{0}=(1-4 / f)^{1 / 2} h$ they have a unique solution, which corresponds to a nearly Kähler structure on $E^{5} \times\left(s_{0}-\varepsilon, s_{0}+\varepsilon\right)$, for some $\varepsilon>0$.

\section{Invariant structures on the Heisenberg group}

In this section we are going to study the construction described above in the particular case where $Q^{3}$ is the three-dimensional Heisenberg group $H_{3}$. Making specific choices of the forms involved and assuming (4.11)-(4.13), we write the equations in Theorem 5.1 and solve them getting explicit solutions. Finally, Proposition 6.2 proves our solution is general.

Let us consider the Heisenberg group $H_{3}$, i.e. the unipotent Lie group given by the upper triangular real matrices of the form $\left(\begin{array}{lll}1 & a & b \\ 0 & 1 & c \\ 0 & 0 & 1\end{array}\right)$. Its Lie algebra is generated by

$$
E_{0}=\left(\begin{array}{lll}
0 & 1 & 0 \\
0 & 0 & 0 \\
0 & 0 & 0
\end{array}\right), E_{1}=\left(\begin{array}{lll}
0 & 0 & 0 \\
0 & 0 & 1 \\
0 & 0 & 0
\end{array}\right), E_{2}=\left(\begin{array}{ccc}
0 & 0 & -1 \\
0 & 0 & 0 \\
0 & 0 & 0
\end{array}\right) \text {. }
$$

They satisfy the commutation relations $\left[E_{1}, E_{2}\right]=-E_{0},\left[E_{0}, E_{1}\right]=\left[E_{0}, E_{2}\right]=0$. If $\sigma_{i}$ is the dual of $E_{i}$, then we have

$$
\mathrm{d} \sigma_{0}=\sigma_{1} \wedge \sigma_{2}, \quad \mathrm{~d} \sigma_{1}=0, \quad \mathrm{~d} \sigma_{2}=0 .
$$

Define $\alpha_{k}:=f_{k}(s) \sigma_{k}$, and set $g_{U U}(s)=g_{V V}(s)=: h(s)$, and $g_{U V}\left(s_{0}\right)=0$ for some initial data $s_{0} \neq 0$. With this choice, equations (5.3) and (5.4) are automatically fulfilled. Then, according to Theorem 5.1, there exists a $T^{2}$-bundle $E^{5} \rightarrow H_{3}$ with connection one-forms $\vartheta_{1}, \vartheta_{2}$ satisfying

$$
s \mathrm{~d}_{5} \vartheta_{1}=-\frac{3 h}{h^{2}-s^{2}} \alpha_{2} \wedge \alpha_{0}, \quad s \mathrm{~d}_{5} \vartheta_{2}=\frac{3 h}{h^{2}-s^{2}} \alpha_{1} \wedge \alpha_{0} .
$$

Equations $\mathrm{d}_{3} \alpha_{k}=0, k=1,2$, imply that all the coefficients $b_{i j}, c_{i j}$ vanish. Furthermore, we have an algebraic relation among the $f_{k}$ 's given by $f_{0} / f_{1} f_{2}=4 h^{2} /\left(h^{2}-s^{2}\right)$. Then we can compute: $a_{10}=a_{20}=a_{12}=a_{21}=0, a_{11}=a_{22}=8 s / 3\left(h^{2}-s^{2}\right)$, and $h^{\prime}=g_{U U}^{\prime}=$ $g_{V V}^{\prime}=-h / s, g_{U V}^{\prime}=0$. So the following differential equations for $\alpha_{0}, \alpha_{1}, \alpha_{2}, \vartheta_{1}, \vartheta_{2}$ hold:

$$
\alpha_{0}^{\prime}=\frac{4 s}{3\left(h^{2}-s^{2}\right)} \alpha_{0}, \quad \alpha_{k}^{\prime}=\frac{8 s}{3\left(h^{2}-s^{2}\right)} \alpha_{k}, \quad \vartheta_{k}^{\prime}=\frac{5}{3\left(h^{2}-s^{2}\right)} \alpha_{k}, \quad k=1,2 .
$$

Since $h=g_{U U}>0$, we obtain the expression $h(s)=\left|s_{0} h\left(s_{0}\right)\right| /|s|=: C / s$, and the following differential equations for $f_{0}, f_{1}, f_{2}$ :

$$
f_{0}^{\prime}=-f_{0}\left(\frac{4 s}{3\left(h^{2}-s^{2}\right)}\right), \quad f_{k}^{\prime}=-f_{k}\left(\frac{8 s}{3\left(h^{2}-s^{2}\right)}\right), \quad k=1,2 .
$$

Hence one can solve them getting

$$
f_{0}(s)=f_{0}\left(s_{0}\right)\left(\frac{C^{2}-s^{4}}{C^{2}-s_{0}^{4}}\right)^{1 / 3}, \quad f_{k}(s)=f_{k}\left(s_{0}\right)\left(\frac{C^{2}-s^{4}}{C^{2}-s_{0}^{4}}\right)^{2 / 3}, \quad k=1,2 .
$$


Let us set $f_{0}\left(s_{0}\right)=f_{1}\left(s_{0}\right)=f_{2}\left(s_{0}\right)=f\left(s_{0}\right)^{-1}$. In this case, having the expressions of $h, f_{0}, f_{1}, f_{2}$, we can write equations (4.1)-(4.4) explicitly: for $0 \neq s^{2}<|C|$ we obtain

$$
\begin{aligned}
g= & \frac{s^{2}}{9\left(C^{2}-s^{4}\right)} \mathrm{d} s^{\otimes 2}+\frac{C}{s}\left(\vartheta_{1}^{\otimes 2}+\vartheta_{2}^{\otimes 2}\right) \\
& +\frac{s^{2}\left(C^{2}-s_{0}^{4}\right)}{16 C^{4}}\left(\left(\frac{C^{2}-s_{0}^{4}}{C^{2}-s^{4}}\right)^{1 / 3} \sigma_{0}^{\otimes 2}+\frac{C}{s}\left(\frac{C^{2}-s^{4}}{C^{2}-s_{0}^{4}}\right)^{1 / 3}\left(\sigma_{1}^{\otimes 2}+\sigma_{2}^{\otimes 2}\right)\right) \\
\sigma= & \frac{s^{2}}{12 C^{2}}\left(\frac{C^{2}-s_{0}^{4}}{C^{2}-s^{4}}\right)^{2 / 3} \mathrm{~d} s \wedge \sigma_{0}+s \vartheta_{1} \wedge \vartheta_{2} \\
+ & \frac{C^{2}-s_{0}^{4}}{4 C^{2}}\left(\frac{C^{2}-s^{4}}{C^{2}-s_{0}^{4}}\right)^{2 / 3}\left(\vartheta_{2} \wedge \sigma_{1}-\vartheta_{1} \wedge \sigma_{2}\right)-\frac{s^{3}\left(C^{2}-s_{0}^{4}\right)}{16 C^{4}}\left(\frac{C^{2}-s^{4}}{C^{2}-s_{0}^{4}}\right)^{1 / 3} \sigma_{1} \wedge \sigma_{2}, \\
\psi_{+}= & \frac{1}{3} \mathrm{~d} s \wedge\left(\vartheta_{1} \wedge \vartheta_{2}+\frac{s^{3}}{4 C^{2}}\left(\frac{C^{2}-s_{0}^{4}}{C^{2}-s^{4}}\right)^{1 / 3}\left(\vartheta_{1} \wedge \sigma_{2}-\vartheta_{2} \wedge \sigma_{1}\right)\right) \\
& -\frac{C^{2}-s_{0}^{4}}{16 C^{4}}\left(\frac{s^{2}}{3}\left(\frac{C^{2}-s^{4}}{C^{2}-s_{0}^{4}}\right)^{1 / 3} \mathrm{~d} s \wedge \sigma_{1} \wedge \sigma_{2}+C s\left(\vartheta_{1} \wedge \sigma_{1}+\vartheta_{2} \wedge \sigma_{2}\right) \wedge \sigma_{0}\right), \\
\psi_{-}= & \frac{s}{12 C}\left(\frac{C^{2}-s_{0}^{4}}{C^{2}-s^{4}}\right)^{1 / 3} \mathrm{~d} s \wedge\left(\vartheta_{1} \wedge \sigma_{1}+\vartheta_{2} \wedge \sigma_{2}\right)+\frac{C^{2}-s_{0}^{4}}{4 C^{2}}\left(\frac{C^{2}-s^{4}}{C^{2}-s_{0}^{4}}\right)^{1 / 3} \vartheta_{1} \wedge \vartheta_{2} \wedge \sigma_{0} \\
& +\frac{s^{2}\left(C^{2}-s_{0}^{4}\right)}{16 C^{4}}\left(s\left(\vartheta_{1} \wedge \sigma_{2}-\vartheta_{2} \wedge \sigma_{1}\right)-\frac{\left(C^{2}-s_{0}^{4}\right)^{1 / 3}\left(C^{2}-s^{4}\right)^{2 / 3}}{4 C^{2}} \sigma_{1} \wedge \sigma_{2}\right) \wedge \sigma_{0} .
\end{aligned}
$$

Remark 6.1. By the expression of the metric $g$ in (6.1) we can observe that the fiber blows up when $s \rightarrow 0$, whereas the remaining four-dimensional subspace collapses to a point. If $s^{2} \rightarrow|C|$, the fiber stabilises, a two-dimensional subspace of the base space collapses to a point, and the rest blows up.

The following proposition states this is indeed a general solution.

Proposition 6.2. The nearly Kähler structure in (6.1)-(6.4) gives a general left-invariant structure for the case of the Heisenberg group $\mathrm{H}_{3}$.

Proof. As seen in Remark 4.3, the geometry of $Q^{3}$ can be described by three one-forms $\beta_{0}, \beta_{1}, \beta_{2}$ satisfying

$$
\mathrm{d} \beta_{0}=\frac{1}{f} \beta_{1} \wedge \beta_{2}, \quad \mathrm{~d} \beta_{1} \wedge \beta_{0}=0, \quad \mathrm{~d} \beta_{2} \wedge \beta_{0}=0 .
$$

Denote by $\tau_{0}, \tau_{1}, \tau_{2}$ a dual basis of the Lie algebra of $H_{3}$ satisfying $\mathrm{d} \tau_{0}=\tau_{1} \wedge \tau_{2}$ and $\mathrm{d} \tau_{1}=\mathrm{d} \tau_{2}=0$. In our particular case we can observe that $\mathrm{d} \beta_{0} \in \operatorname{Span}\left\{\tau_{1} \wedge \tau_{2}\right\}$, so $\beta_{1} \wedge \beta_{2}=c_{1} \tau_{1} \wedge \tau_{2}$ for some real number $c_{1} \neq 0$. This happens only when $\beta_{1}, \beta_{2} \in$ $\operatorname{Span}\left\{\tau_{1}, \tau_{2}\right\}$, so $\mathrm{d} \beta_{1}=\mathrm{d} \beta_{2}=0$, and then $\mathrm{d} \beta_{1} \wedge \beta_{0}=\mathrm{d} \beta_{2} \wedge \beta_{0}=0$, as we wanted.

Therefore $\beta_{0}=\frac{c_{1}}{f} \tau_{0}+a \tau_{1}+b \tau_{2}$ for some real numbers $a, b$. Now define $\sigma_{0}:=f \beta_{0}$, and choose $\sigma_{1}, \sigma_{2} \in \operatorname{Span}\left\{\tau_{1}, \tau_{2}\right\}$ so that $\sigma_{1}$ is $\tilde{g}$-orthogonal to $\sigma_{2},\left\|\sigma_{1}\right\|_{\tilde{g}}=\left\|\sigma_{2}\right\|_{\tilde{g}}, \sigma_{1} \wedge \sigma_{2}=$ $\beta_{1} \wedge \beta_{2}$, and $\sigma_{1} \wedge \sigma_{2}=c_{2} \tau_{1} \wedge \tau_{2}$, for some positive constant $c_{2}$. Define $\tilde{\beta}_{0}:=\beta_{0}, \tilde{\beta}_{1}:=\sigma_{1}$, and $\tilde{\beta}_{2}:=\sigma_{2}$. This new dual frame satisfies

$$
\mathrm{d} \tilde{\beta_{0}}=\frac{1}{f} \tilde{\beta}_{1} \wedge \tilde{\beta_{2}}, \quad \mathrm{~d} \tilde{\beta}_{1}=\mathrm{d} \tilde{\beta}_{2}=0, \quad \tilde{g}=h \mathrm{Id},
$$


As in the end of Section 4, we can define three one-forms $\tilde{\alpha}_{i}, i=0,1,2$ such that $\tilde{\beta}_{0}=: \tilde{\alpha}_{0}$ and $\tilde{\beta}_{i}=: f \tilde{\alpha}_{i}, i=1,2$. Hence

$$
\begin{aligned}
& \tilde{\alpha}_{0}\left(s_{0}\right)=\tilde{\beta}_{0}\left(s_{0}\right)=\frac{1}{f\left(s_{0}\right)} \sigma_{0} \\
& \tilde{\alpha}_{k}\left(s_{0}\right)=\frac{1}{f\left(s_{0}\right)} \tilde{\beta}_{k}\left(s_{0}\right)=\frac{1}{f\left(s_{0}\right)} \sigma_{k}, \quad k=1,2,
\end{aligned}
$$

so if $f_{0}, f_{1}, f_{2}$ are such that $\tilde{\alpha}_{k}=f_{k}(s) \sigma_{k}$, we get $f_{0}\left(s_{0}\right)=f_{1}\left(s_{0}\right)=f_{2}\left(s_{0}\right)=f\left(s_{0}\right)^{-1}$. Thus it is always possible to restrict ourselves to the case studied above.

\section{References}

[1] Christian Bär, Real Killing spinors and holonomy, Comm. Math. Phys. 154 (1993), no. 3, 509-521.

[2] Jean-B. Butruille, Classification des variétés approximativement kähleriennes homogènes, Ann. Global Anal. Geom. 27 (2005), no. 3, 201-225.

[3] Ramón R. Carrión, Some special geometries defined by Lie groups, Ph.D. thesis, Oxford, 1993.

[4] Lorenzo Foscolo and Mark Haskins, New $G_{2}$-holonomy cones and exotic nearly Kähler structures on $S^{6}$ and $S^{3} \times S^{3}$, Ann. of Math. 185 (2017), no. 1, 59-130.

[5] Alfred Gray, Nearly Kähler manifolds, J. Differential Geom. 4 (1970), no. 3, 283-309.

[6] — The structure of nearly Kähler manifolds, Math. Ann. 223 (1976), 233-248.

[7] Shoshichi Kobayashi and Katsumi Nomizu, Foundations of differential geometry, vol. 1,2, Wiley Classics Library, 1996.

[8] Thomas B. Madsen and Andrew F. Swann, Multi-moment maps, Adv. Math. 229 (2012), 2287-2309.

[9] Andrei Moroianu, Paul-Andi Nagy, and Uwe Semmelmann, Deformations of nearly Kähler structures, Pacific J. Math. 235 (2008), no. 1, 57-72.

[10] Paul-Andi Nagy, On nearly-Kähler geometry, Ann. Global Anal. Geom. 22 (2002), $167-178$.

[11] Andrew F. Swann, Twisting Hermitian and hypercomplex geometries, Duke Math. J. 155 (2010), no. 2, 403-431.

(G. Russo) Department of Mathematics and Centre for Quantum Geometry of Moduli Spaces, Aarhus University, Ny Munkegade 118, Bldg 1530, DK-8000 Aarhus C, Denmark E-mail address: giovanni.russo@math.au.dk

(A. F. Swann) Department of Mathematics, Centre for Quantum Geometry of Moduli Spaces, Aarhus University, and Aarhus University Centre for Digitalisation, Big Data and Data Analytics, Ny Munkegade 118, Bldg 1530, DK-8000 Aarhus C, Denmark

E-mail address: swann@math.au.dk 